\title{
Novel injectable gel (system) as a vehicle for human articular chondrocytes in cartilage tissue regeneration
}

\author{
R. C. Pereira ${ }^{1,3,4}$, M. Scaranari ${ }^{1}$, P. Castagnola ${ }^{2}$, M. Grandizio ${ }^{5}$, H. S. Azevedo ${ }^{3,4}$, R. L. Reis ${ }^{3,4}$, \\ R. Cancedda ${ }^{1,2}$ and C. Gentili ${ }^{1,2 *}$ \\ ${ }^{1}$ Dipartimento di Biologia, Oncologia e Genetica, University of Genova, Italy \\ ${ }^{2}$ Istituto Nazionale per la Ricerca sul Cancro, Genova, Italy \\ ${ }^{3} 3$ Bs Research Group - Biomaterials, Biodegradables and Biomimetics, University of Minho, Headquarters of the European Institute of \\ Excellence on Tissue Engineering and Regenerative Medicine, AvePark, 4806-909 Taipas, Guimarães, Portugal \\ ${ }^{4} I B B$ - Institute for Biotechnology and Bioengineering, PT Associated Laboratory, Guimarães, Portugal \\ ${ }^{5}$ Ospedale S. Antonio Recco, ASL 3 Genovese, Genova, Italy
}

\begin{abstract}
We developed a novel injectable carrageenan/fibrin/hyaluronic acid-based hydrogel with in situ gelling properties to be seeded with chondrogenic cells and used for cartilage tissue engineering applications. We first analysed the distribution within the hydrogel construct and the phenotype of human articular chondrocytes (HACs) cultured for 3 weeks in vitro. We observed a statistically significant increase in the cell number during the first 2 weeks and maintenance of cell viability throughout the cell culture, together with the deposition/formation of a cartilage-specific extracellular matrix (ECM). Taking advantage of a new in vivo model that allows the integration between newly formed and preexisting cartilage in immunodeficient mice to be investigated, we showed that injectable hydrogel seeded with human articular chondrocytes was able to regenerate and repair an experimentally made lesion in bovine articular cartilage, thus demonstrating the potential of this novel cell delivery system for cartilage tissue engineering. Copyright $\odot 2009$ John Wiley \& Sons, Ltd.
\end{abstract}

Received 27 October 2008; Accepted 12 December 2008

Keywords cartilage tissue engineering; cell culture; biomaterial; animal model; extracellular matrix (ECM)

\section{Introduction}

Tissue loss, as a result of trauma, congenital disorders and joint diseases, usually involving structural damage of articular cartilage surface, is a relevant clinical problem (Cancedda et al., 2003; Tuli et al., 2003). In fact these pathologies result in severe pain and disability for millions of people worldwide and represent a major challenge for orthopaedic surgeons (Hunziker, 1999, 2000; Temenoff and Mikos, 2000b; Giannoni and Cancedda, 2006). Tissue engineering (TE) can be defined as the art

*Correspondence to: C. Gentili, Istituto Nazionale per la Ricerca sul Cancro, Largo Rosanna Benzi 10. I-16132 Genova, Italy. E-mail: chiara.gentili@istge.it of reconstructing tissues (Cancedda et al., 2003), both structurally and functionally, with the knowledge that reconstruction can be performed either entirely or partially in vitro and then completed in vivo (Temenoff and Mikos, 2000b; McHale et al., 2005; Ando et al., 2007; Endres et al., 2007; Eyrich et al., 2007a, 2007b).

During the past decade exciting new tissue-engineering strategies have emerged that show the potential to regenerate damaged cartilage (Hunziker, 2000; Temenoff and Mikos, 2000b; Gelse and Schneider, 2006). In particular, autologous chondrocyte transplantation (ACT), a standard clinical procedure to treat human articular cartilage defects (Brittberg et al., 1994; Peterson et al., 2000), has been successfully used on thousands of patients. However, ACT, in addition to some intrinsic limitations, such 
as a poor in vitro expansion of chondrocytes and some complexity in the graft fixation, requires an invasive surgical technique. To overcome some of these problems, new materials and new models have been studied and proposed as cell delivery systems (Temenoff and Mikos, 2000b; Lee and Mooney, 2001; Chung and Burdick, 2008).

A very new and exciting approach to cell delivery for tissue engineering is using hydrogels, which have the ability to be injected into the body as a solution and to form a gel immediately after injection, thus enabling the clinician to transplant the cell-support system in a minimally invasive manner. The use of materials that present an injectable formulation satisfies also the need of a perfect filling of the lesion, as they can also be easily applied in the presence of an irregular defect (Gutowska et al., 2001; Lee and Mooney, 2001; Park et al., 2007).

Biodegradable and biocompatible hydrogels with components and structure similar to the extracellular matrices (ECM) present in different districts of the body have found numerous applications in the fields of TE and in drug delivery (Temenoff and Mikos 2000a; Park et al., 2005; DeFail et al., 2006; Endres et al., 2007; Eyrich et al., 2007b; Park et al., 2007). In particular, several scaffolds/hydrogels have been proposed in association with different cell types for articular cartilage regeneration (Temenoff and Mikos, 2000a; Gutowska et al., 2001; Cancedda et al., 2003; Neuss et al., 2008). Although the use of synthetic hydrogels appears very promising, there are some important aspects that must be investigated before they can be widely used in clinical practice (Cancedda et al., 2003; Chung and Burdick, 2008). More specifically, the interactions of the cells with the hydrogels can interfere with cell adhesion, as well as with cell proliferation and migration, the adhesion being cell-type specific and dependent on the interactions of specific cell receptors with components adsorbed or encapsulated into the materials (e.g. ECM macromolecules and growth factors).

The aim of this study was to investigate some of these aspects in the case of a new biodegradable carrageenanbased injectable gel. Recent papers described a new organ culture model in which human articular chondrocytes were cultured within an experimentally made osteochondral defect in an articular cartilage biopsy subcutaneously implanted in an immunodeficient mouse (Mueller-Rath et al., 2007; Schuller et al., 2008). We adopted a similar approach to show that carrageenan-based hydrogel, seeded with human articular chondrocytes (HACs), has the potential to regenerate and repair an articular defect.

\section{Materials and methods}

\subsection{Hydrogel preparation}

$\kappa$ - and $\iota$-carrageenans (Sigma-Aldrich, Germany) were dissolved in double-distilled water $(0.8 \%$ and $1.2 \%$ $\mathrm{w} / \mathrm{v}$, respectively) and the solution was heated at $65^{\circ} \mathrm{C}$ under constant stirring to obtain a final homogeneous preparation. Carrageenans are high molecular weight sulphated polygalactans derived from several species of red seaweeds (Rhodophyceae). The most common forms of carrageenan are lambda $(\lambda)$, kappa $(\kappa)$ and iota $(\iota)$. Carrageenan has alternating disaccharide units composed of D-galactose-2-sulphate and D-galactose-2,6-disulphate, the galactose residues being joined by $-1,3$ and $-1,4$ linkages. These polysaccharides form gels upon cooling in the presence of specific ions, due to the development of $3 \mathrm{D}$ networks as the result of the formation of helical chain complexes. In the presence of $\mathrm{K}^{+}$ions, $\kappa$-carrageenan forms strong crisp gels, whereas $\iota$-carrageenan in the presence of $\mathrm{Ca}^{2+}$ ions forms elastic gels (Bartkowiak and Hunkeler, 2001; Thommes and Kleinebudde, 2008).

Fibrinogen (final concentration, 3000 KalliKreininhibitor-units (KIU/ml); Baxter AG, Austria) and thrombin (final concentration, $500 \mathrm{U} / \mathrm{ml}$; Baxter) were dissolved in an aprotinin solution (10000 KalliKreininhibitor-units (KIU/ml; Baxter) and in $40 \mathrm{mM} \mathrm{CaCl}_{2}$, respectively. Human chondrocytes, harvested from a human knee biopsy (Figure 1A, B) and cultured in serumfree medium (see following paragraph) were resuspended at a concentration of $2 \times 10^{6}$ cells $/ \mathrm{ml}$ in culture medium (Biochrom AG, Berlin, Germany) containing $0.265 \mathrm{~g} / 1$ $\mathrm{CaCl}_{2}$ and $0.4 \mathrm{~g} / 1 \mathrm{KCl}$ in order to trigger gel formation when mixed with the carrageenan polymeric solution. The cell suspension was supplemented with the thrombin solution $(5.9 \% \mathrm{v} / \mathrm{v})$ and with $0.75 \% \mathrm{v} / \mathrm{v}$ hyaluronic acid before being loaded into syringe a of the injection system (Figure 1C). Syringe b was loaded with the carrageenan solution supplemented with the fibrinogen solution at $35 \% \mathrm{v} / \mathrm{v}$. The carrageenan/fibrinogen solution was mixed with the cells/thrombin/hyaluronic acid solution by activating the two syringes at the same time. The gel formed almost instantaneously.

\subsection{In vitro cell culture}

Articular cartilage biopsies were collected from male or female patients (aged 61-75 years) undergoing total knee arthroplasty. Patients had signed an informed consent document approved by the Ethical Committee of the San Antonio Hospital, Recco, Italy.

Briefly, cartilage was cleaned of connective tissue and/or subchondral bone and cut into small fragments, according to published procedures (Malpeli et al., 2004). Individual HACs were released by repeated enzymatic digestions with collagenase I $(400 \mathrm{U} / \mathrm{ml})$, collagenase II $(1000 \mathrm{U} / \mathrm{ml}$; Worthing Biochemical, Lakewood, NJ, USA), hyaluronidase ( $1 \mathrm{mg} / \mathrm{ml}$; Sigma, St. Louis, MO, USA) and $0.25 \%$ trypsin (Invitrogen Life Technologies, Carlsbad, CA, USA) at $37^{\circ} \mathrm{C}$. Cells were pooled, counted and seeded in culture dishes precoated for $24 \mathrm{~h}$ with $10 \%$ fetal calf serum (FCS) in culture medium (Biochrom A.G. Berlin, Germany) to allow attachment of the serum fibronectin to the plastic dish surface. After $48 \mathrm{~h}$ from seeding, the chondrocytes were enzymatically 


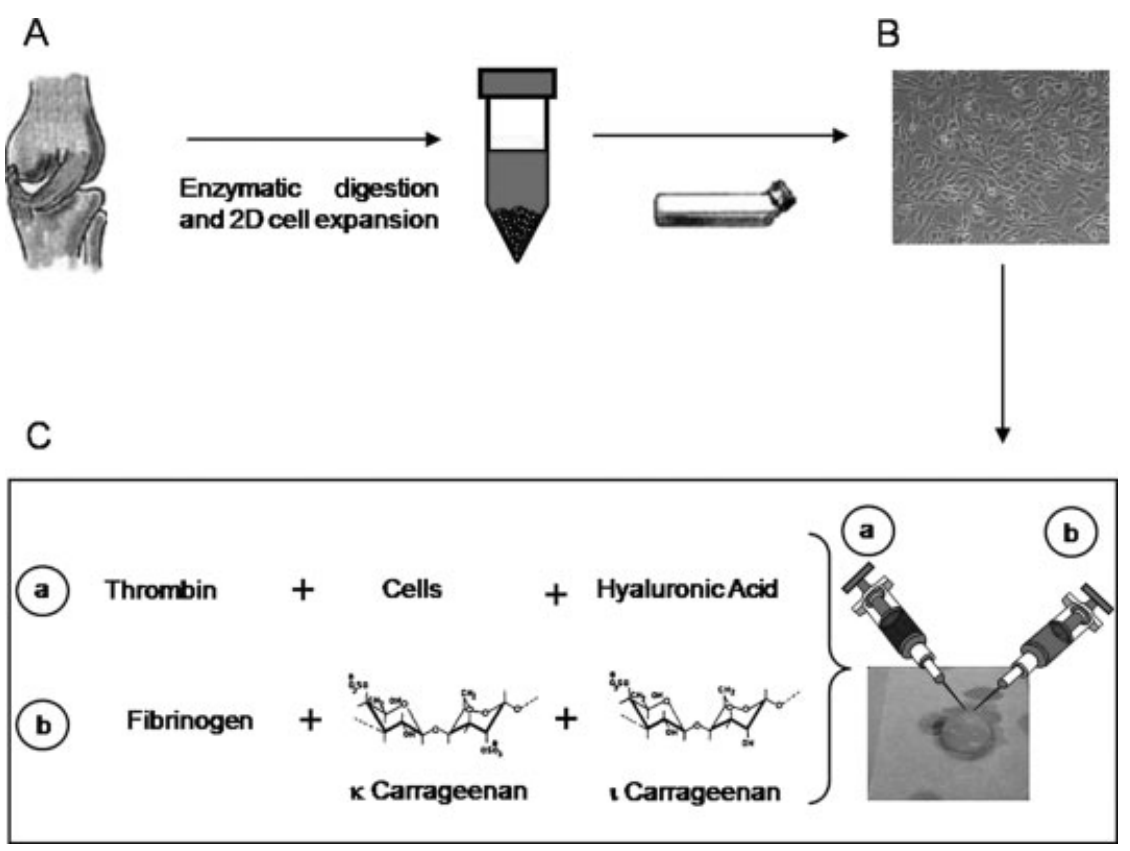

Figure 1. Scheme of the procedure followed to form the hydrogel. (A) Human articular chondrocytes biopsy and digestions. (B) Light microscopy of a primary monolayer culture in serum-free (SF) medium before the recovery of the in vitro expanded cells. (C) Biodegradable hydrogel system: content composition in syringes $a$ and $b$ and gross morphology of the formed gel

detached, collected, washed in PBS, counted and replated in dishes pre-rinsed several times with PBS to remove any residual trace of serum. The culture medium was then replaced with the serum-free (SF) medium described by Malpeli et al. (2004) and the cells expanded in vitro in this culture condition. The medium was changed three times/week. After four to five cell doublings, HACs were collected and either encapsulated within the tissue engineering construct and the culture continued in chondrogenic medium (Johnstone et al., 1998) for 3 weeks, or pelleted by centrifugation $(2.5 \times$ $10^{5}$ cells) and cultured in chondrogenic medium for 3 weeks.

\subsection{Cell imaging}

Over the course of the culture, the engineered constructs $(n=3)$ were monitored using a brightfield light microscope (Axiovert 10, Zeiss, Germany) equipped with a digital camera (Olympus DP 10, Olympus Optical, Japan). Images were acquired at $\times 5$ and $\times 10$ magnifications.

To assess live cells distribution, the constructs were incubated with the nuclear staining Hoescht 33342 (Sigma-Aldrich, Germany) at a final concentration of $5 \mu \mathrm{g} / \mathrm{ml}$ in culture medium for $30 \mathrm{~min}$.

Optical sections along the $z$ axis were then acquired by structured epifluorescent illumination, using an Axiovert 200M microscope equipped with the Apotome module, filter set 49 and an AxiocamHR camera (Carl Zeiss, Jena, Germany). Image acquisition stacking of the optical sections and $3 \mathrm{D}$ reconstruction of the samples were performed using Axiovision Software (Carl Zeiss) (Frey et al., 2006; Osakada et al., 2008).

\subsection{MTT analysis}

The viability of encapsulated HAC within the hydrogel was evaluated at different time intervals, using thiazolyl blue staining (MTT, Sigma-Aldrich), in which absorbance data correlate to cell number. Briefly, in triplicate sample culture, at each time point the medium was removed and replaced with $0.5 \mathrm{ml}$ fresh serum-free medium supplemented with $25 \mu \mathrm{l}$ MTT stock solution $(5 \mathrm{mg} / \mathrm{ml})$. After $3 \mathrm{~h}$ incubation, the medium was collected and the converted dye was solubilized with $1 \mathrm{ml}$ absolute ethanol. Dye absorbance was measured at $570 \mathrm{~nm}$ with background subtraction at $670 \mathrm{~nm}$ (Denizot and Lang, 1986).

\subsection{Semi-quantitative reverse transcriptase-polymerase chain reaction (RT-PCR)}

RNA was extracted using the TRIzol ${ }^{\circledR}$ reagent (Invitrogen, Carlsbad CA, USA) procedure (Ulivi et al., 2006). Conditions for semi-quantitative RT-PCR are described in Banfi et al. (2000). The mRNAs analysed were aggrecan, $\alpha 1$ (I) collagen (collagen type I), $\alpha 1$ (II) collagen (collagen type II) and glyceraldehyde-3-phosphate dehydrogenase $(G A P D H)$. Primer sequences were as follows:

Aggrecan: forward, 5'-ATGCCCAAGACTACCAGTGG - 3', reverse, 5'-GTGAGCTCCGCTTCTGTAGT-3'

Collagen type $I$ : forward, 5'-TCTGCGACACAAGGAGTCTG $-3^{\prime}$, reverse, 5'-CGACCCACACTTCCATCACT-3' Collagen type II: forward, 5'-TCTGCAACATGGAGACTAGC-3', reverse, 5'-GAAGCAGACAGGCCCTATGT-3' 
GAPDH: forward, 5'-CCATCTTCCAGGAGCGAGAT-3', reverse, 5'-CTGCTTCACCACCTTCTTGAT-3'

Following a $4 \mathrm{~min}$ denaturation step at $95^{\circ} \mathrm{C}$, the adopted reaction profiles were: aggrecan, collagen type $I$ and $G A P D H, 95^{\circ} \mathrm{C}$ for $1 \mathrm{~min}, 55^{\circ} \mathrm{C}$ for $1 \mathrm{~min}$ and $72^{\circ} \mathrm{C}$ for $1 \mathrm{~min}$ for 25 cycles; collagen type II, $95^{\circ} \mathrm{C}$ for $1 \mathrm{~min}$, $60^{\circ} \mathrm{C}$ for $1 \mathrm{~min}$ and $72^{\circ} \mathrm{C}$ for $1 \mathrm{~min}$ for 30 cycles. All PCRs ended with $8 \mathrm{~min}$ incubation at $72{ }^{\circ} \mathrm{C}$. The values of the cycles used were in the linear range of amplification, as determined previously during preliminary experiments. PCR products were run on $1 \%$ agarose gels and visualized using ethidium bromide. Reaction product sizes were: aggrecan, 448 bp; collagen type I, 599 bp; collagen type II, $517 \mathrm{bp}$ and $G A P D H, 450 \mathrm{bp}$.

\subsection{Bovine organ culture defect model}

Bovine articular cartilage was harvested from the knees of 18 month-old cows, washed twice with PBS and cut into regular cubes of about $1.5 \times 1.5 \times 0.5 \mathrm{~cm}$. Cartilage defects were created on the cartilage surface using a drilling device $(0.4 \mathrm{~cm})$ under sterile conditions. The defects were then filled with hydrogel seeded or not seeded with $2 \times 10^{6} / \mathrm{ml}$ cells. Six engineered cartilage pieces were subsequently implanted subcutaneously in immunodeficient mice $(\mathrm{nu} / \mathrm{nu})$ and the animals were sacrificed after 4, 6 and 8 weeks to recover the cartilage pieces. All animals were treated and handled according to institutional guidelines.

\subsection{Histology and immunohistochemistry}

Histological samples were rinsed three times in PBS and then fixed in $4 \%$ formaldehyde in PBS for $6 \mathrm{~h}$. After fixation, the samples were dehydrated by serial immersion in ethanol solutions (70\%, 90\%, 95\% and 100\%) and xylene. Specimens were then embedded in paraffin and $5 \mu \mathrm{m}$ sections were cut using a microtome (Leica RM 2165). Sections were then dewaxed and stained with toluidine blue (Merck, Darmstadt, Germany), or Alcian blue (Sigma-Aldrich Chemical, St. Louis, MO, USA) to reveal cartilage matrix components.

For immunohistochemical analysis, serial sections of paraffin-embedded samples were dewaxed and treated with methanol: $\mathrm{H}_{2} \mathrm{O}_{2}(49: 1)$ for $30 \mathrm{~min}$ to inhibit endogenous peroxidases. The sections were then treated with $1 \mathrm{mg} / \mathrm{ml}$ hyaluronidase in PBS, pH 6.0, for $20 \mathrm{~min}$ at $37^{\circ} \mathrm{C}$ and washed with PBS. After incubation with goat serum for $1 \mathrm{~h}$ to reduce non-specific binding, the specific antibody was added and incubated for $1 \mathrm{~h}$ at room temperature. Sections were washed several times in PBS and challenged with biotinylated anti-mouse IgG (Jackson Laboratory Inc., West Grove, PA, USA) and peroxidaseconjugated egg-white avidin (Jackson Laboratory). After additional washing of the sections with PBS and $50 \mathrm{~mm}$ sodium acetate, $\mathrm{pH} 5.0$, the peroxidase activity was visualized by enzymatic modification of the 3-amino9-ethylcarbazole substratum (3-amino-9-ethylcarbazole $0.4 \%$ in dimethylformamide:50 mM sodium acetate, $\mathrm{pH}$ $5: 30 \% \mathrm{H}_{2} \mathrm{O}_{2} ; 100: 900: 1$ ) performed in the dark at room temperature for $15 \mathrm{~min}$. Sections were counterstained with Harri's haematoxylin and mounted with Gel Mount (Biomeda Corp., Foster City, CA, USA). The slides were observed and images acquired with the Axiovert 200M microscope (Carl Zeiss).

The specific antibodies were an anti-type II collagen monoclonal antibody (CIICI anti-COLLII, DSHB, University of Iowa) and anti-human Tenascin monoclonal antibody (a generous gift from Dr E. Balza, Istituto Nazionale per la Ricerca sul Cancro, Genova).

\subsection{Statistical analysis}

All data were analysed by one-way ANOVA, followed by a post hoc comparison with Turkey's HDS. Differences were accepted to be statistically significant at $p<0.05$. All errors are given as standard deviations.

\section{Results}

\subsection{In vitro culture of articular chondrocytes seeded into the injectable hydrogel}

Human articular chondrocytes were expanded up to a maximum of five doublings in serum-free medium. This culture condition also allows the proliferation of chondrocytes derived from elderly donors, maintaining at the same time the cell chondrogenic potential needed for therapeutic application (Dozin et al., 2002).

Cells grown in monolayer were detached and resuspended at a concentration of about $4 \times 10^{6} / \mathrm{ml}$ in a solution containing hyaluronic acid and thrombin and loaded into syringe a of a Duploject syringe support (included in the Tissucol Kit, Baxter). Syringe b was loaded with a solution containing fibrinogen and $\kappa$ - and ı-carrageenan. Upon mixing, the two solutions almost instantaneously formed a gel containing the cells; for each sample, the total volume of the gel was about $250 \mu$ l. Chondrocytes embedded in this gel were cultured in $1.5 \mathrm{ml}$ chondrogenic medium (Johnstone et al., 1998) for 3 weeks.

Cell morphology and distribution within the gel were examined by microscopy at several time points during in vitro culture. Immediately after the hydrogel formation, the cells appeared round and homogeneously distributed, but during the first week of culture they showed a tendency to form clusters (Figure 2A, B). After 3 weeks we observed a transition of the encapsulated chondrocytes from an aggregate status to a more homogeneous and uniform distribution (Figure 2D, E). Optical sections of the specimens incubated with Hoechst dye and subsequent 3D reconstruction allowed the identification and the visualization of the cells yielding, an accurate 
3D distribution of these within the scaffolds (Figure 3). Distribution of human chondrocytes over the total depth of the gel was observed after both 1 and 3 weeks of culture (Figure 3).

Viability of encapsulated cells within the polysaccharide biodegradable hydrogel during the 3 weeks of culture was confirmed using MTT analysis. The number of cells increased during the initial 2 weeks of culture
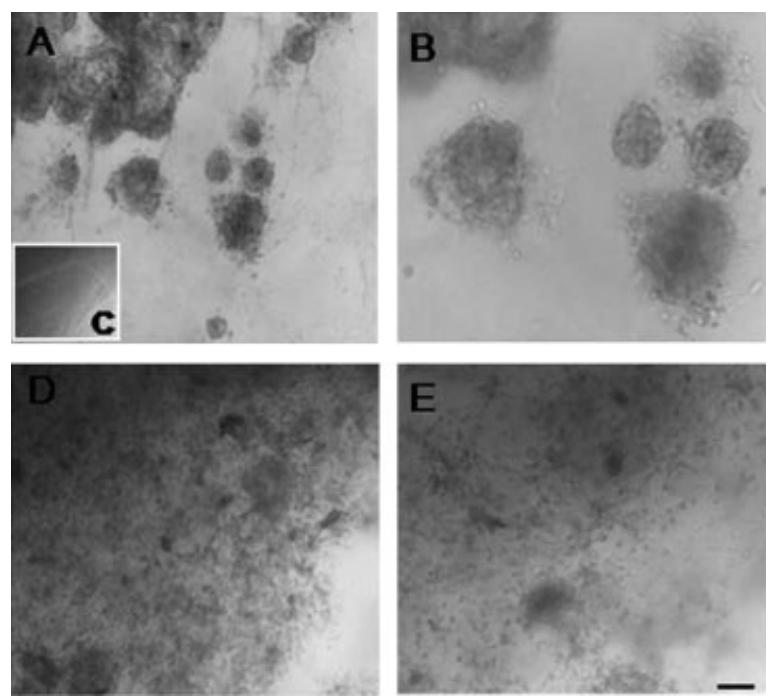

Figure 2. Light microscopy images of the hydrogel containing the human chondrocytes after 1 week (A, B) and 3 weeks (D, E) of culture. A control hydrogel not seeded with cells after 1 week of culture is shown in (C). Bar $=50 \mu \mathrm{m}$
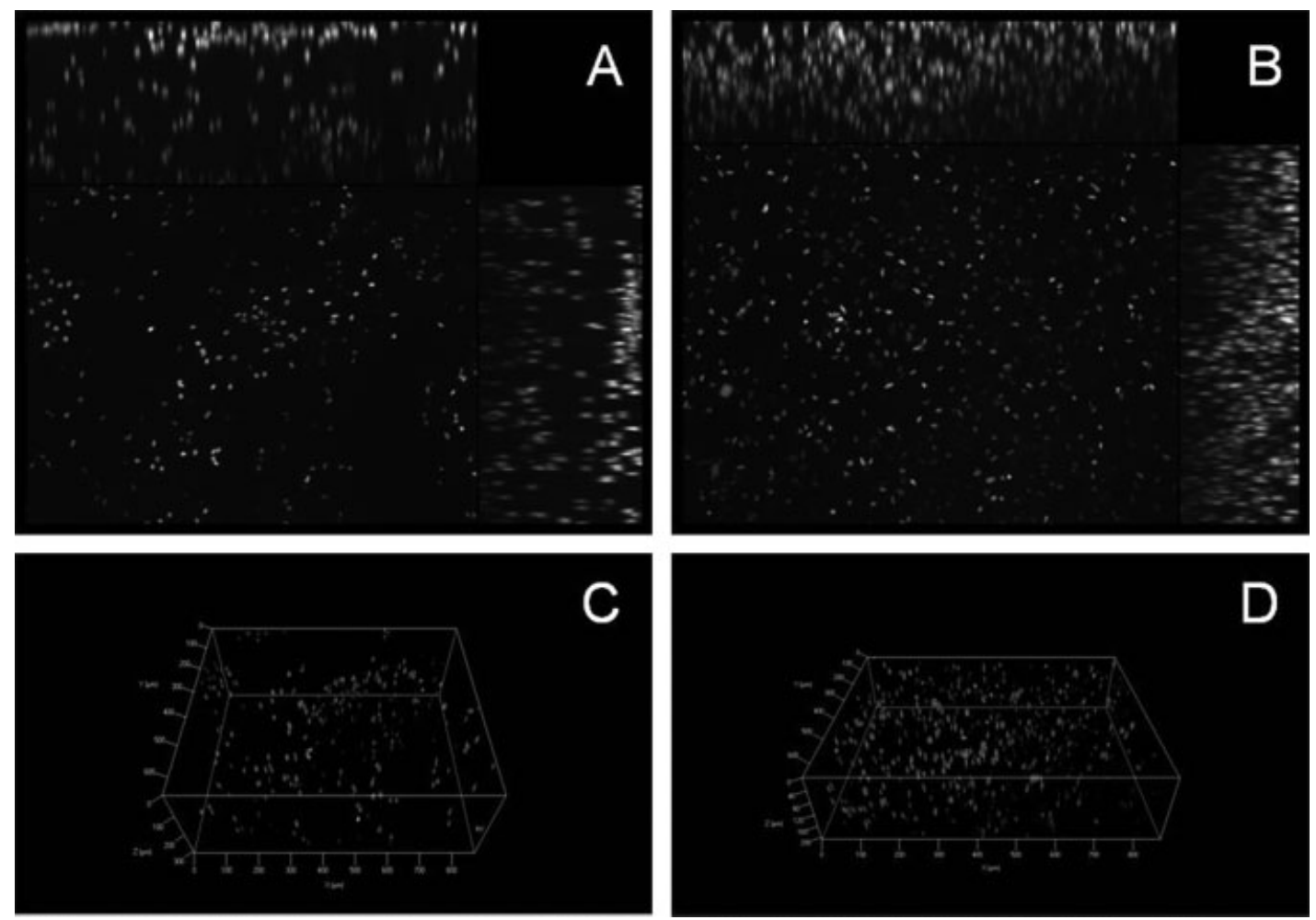

Figure 3. Representative images of live human articular chondrocytes within the tissue-engineered constructs at 1 (A, C) and 3 weeks (B, D) of culture after labeling of the cell nuclei with Hoechst and optical sectioning by structured epifluorescent illumination. Image projections along the $x, y$ and $z$ axes (A, B) and their respective 3D reconstructed stacks (C, D) are shown 


\subsection{In vitro cartilage formation}

To test the capability of the hydrogel embedded cells to form cartilage, human articular chondrocytes expanded in monolayer were seeded into the hydrogel and maintained for 3 weeks in chondrogenic medium. At the end of monolayer culture and before encapsulation into the hydrogel $\left(T_{0}\right)$, human articular chondrocytes expanded in the presence of serum-free medium expressed the chondrocyte-specific aggrecan and type II collagen mRNAs and, as expected, also type I collagen mRNA (Figure 5). After 3 weeks of in vitro culture within the gel $\left(T_{\mathrm{F}}\right)$, the encapsulated human articular chondrocytes maintained the expression of aggrecan and type II collagen but stopped the expression of type I collagen.

As a further control, at the time of the establishment of the culture within the hydrogel, an aliquot of the cells from the monolayer culture was used to establish a parallel in vitro micromass pellet culture (Malpeli et al., 2004) that was maintained in the same chondrogenic medium as the hydrogel culture. Histological analyses were performed for both the hydrogel and the micromass in vitro culture after 3 weeks.

Mucopolysaccharides and glycosaminoglycans, characteristic of the cartilage ECM, revealed by toluidine (Figure 6B, D) and Alcian blue (Figure 6A-C) were detected in the micromass pellet, as described by Johnstone et al. (1998), and in hydrogel culture after 3 weeks. It was noteworthy that the distribution of the cartilage matrix in the hydrogels was more homogeneous than in the micromass pellets, where it was preferentially located at the peripheral regions.

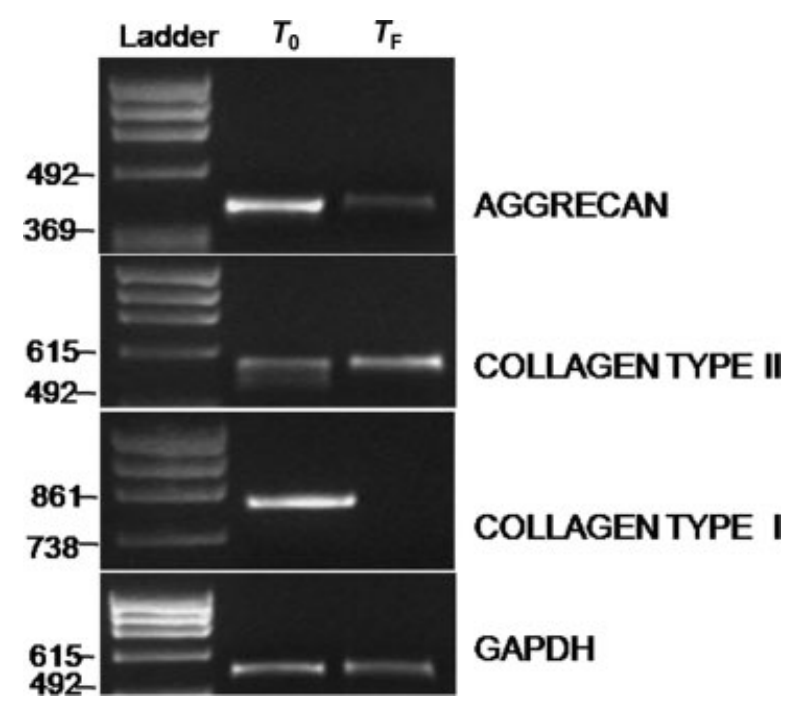

Figure 5. Expression of chondrogenic marker genes by 3D-cultured articular chondrocytes. Reverse-transcribed cDNA was amplified by PCR with specific primers for the housekeeping gene GAPDH (5698 bp), collagen type I (750 bp); collagen type II (517 bp) and aggrecan (448 bp). Ladder, DNA molecular weight markers (in base pairs) is shown on the left side; $T_{0}$, expanded chondrocytes before encapsulation; $T_{\mathrm{F}}$, encapsulated chondrocytes after 3 weeks of in vitro culture

\subsection{In vivo implantation of human articular chondrocytes-hydrogel constructs}

The immunodeficient mouse model is a well-established method to investigate in vivo engineering of cartilage and bone (Hunziker, 1999; Liu and Cao, 2007; MuellerRath et al., 2007). Nevertheless, in the case of cartilage engineering, one of the major limitations of this method is the lack of an appropriate articular cartilage surrounding the newly formed tissue, and therefore the impossibility of investigating the integration of the newly formed cartilage with the pre-existing cartilage. We used an organ culture model, recently described (Mueller-Rath et al., 2007; Schuller et al., 2008), in which the hydrogel, including encapsulated HACs, was directly injected into the defect created on the surface of the bovine articular cartilage layer (Figure 7). The cartilage biopsy with the hydrogel was maintained in culture in chondrogenic medium for 1 week, then implanted subcutaneously in the immunodeficient mouse. Histological analysis was performed on cross-sections of the recovered samples after 4, 6 and 8 weeks of implantation (Figure 8).

Macroscopic inspection of the retrieved implanted construct showed a smooth surface and a complete integration of the HAC-hydrogel with the surrounding cartilage (Figure 8).

Histological examination of the sample revealed the repair of the bovine cartilage defect by a cartilage tissue. The newly formed cartilage showed matrix deposition and uniform cellularity, it was clearly stained by toluidine blue (Figure $8 \mathrm{~A}-\mathrm{C}, \mathrm{E}$ ) and was positive for the presence of type II collagen (Figure $8 \mathrm{~F}$ ). Moreover, at the tissue interface a good integration and grip between the newly formed human cartilage and the surrounding bovine cartilage was observed. In a control biopsy treated with the hydrogel without cells, we failed to detect a newly formed organized cartilage and an integration of the
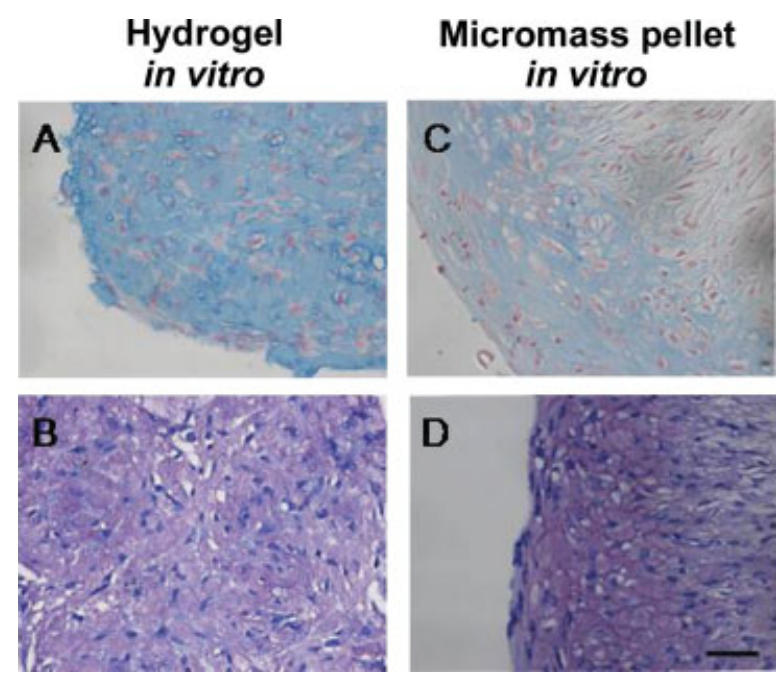

Figure 6. Histological cross-sections of the hydrogel (A, B) and micromass pellet (C, D) after 3 weeks of culture. Sections were stained with Alcian blue (A, C) and toluidine blue (B, D). Bar $=50 \mu \mathrm{m}$ 
A

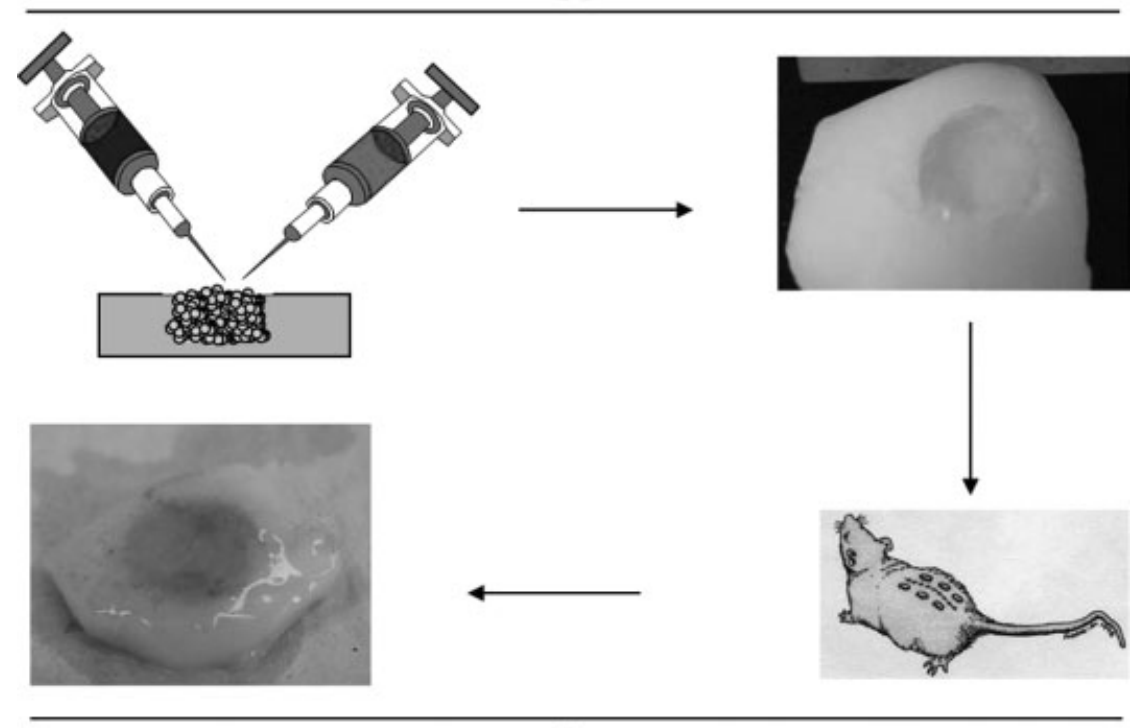

B

Figure 7. Contained-defect organ culture model: bovine cartilage defects filled with hydrogel containing human articular chondrocytes (A); organ construct model implanted for 6 weeks in an immunodeficient mouse (B)

tissue filling the gap with the bovine cartilage (Figure 8D). The origin of the neoformed cartilage was confirmed by immunolocalization of human tenascin, taking advantage of a specific monoclonal antibody which stained the human chondrocytes in the neoformed tissue and not those in the surrounding bovine cartilage (Figure 9A, B).

\section{Discussion}

Cartilage tissue engineering is an emerging approach for the regeneration of cartilage tissue damaged due to disease or trauma. Since cartilage lacks spontaneous regenerative capabilities, it is essential to develop methodologies for the delivery of appropriate cells, biomaterials and factors to the defect site. The articular chondrocyte transplantation technique (Brittberg et al., 1994; Peterson et al., 2000) is a commonly used method for cartilage repair, requiring a first arthroscopic intervention to harvest the healthy cartilage sample, from which chondrocytes are isolated and expanded in vitro. With a second arthrotomy intervention, these cells are then delivered to the cartilage defect site under a periosteal or synthetic membrane flap.

The goal of this study was to determine the possibility of delivering human articular chondrocytes by encapsulating them in a novel, easy-to-handle, biodegradable hydrogel, with in situ gelation properties and not affecting cell chondrogenic differentiation. We developed a suitable injectable biomaterial obtained by the mixture of hyaluronic acid, fibrinogen, thrombin and carrageenan.

Carrageenans are heteropolysaccharides structurally related to agar (Bartkowiak and Hunkeler, 2001). They are extracted from red algae and come in three types, $\kappa$, $\iota$ and $\lambda$, that can form upon cooling a variety of gels. The rationale of using a carrageenan hydrogel, rather than gels made of synthetic polymers or other polysaccharides, was mainly based on the ability of these polysaccharides to form a gel at conditions that allow the direct encapsulation of mammalian cells. These polysaccharides are watersoluble when heated and gel upon cooling the solution in presence of electrolytes $\left(\mathrm{Ca}^{2+}\right.$ and $\mathrm{K}^{+}$) (Eyrich et al., 2007b). In the conditions described in our study, we were able to mix the cell suspension with the polysaccharide solution and then form a gel at temperatures in the range $36-40^{\circ} \mathrm{C}$ with retention of cell viability. $\kappa$ - and $\iota$-carrageenan are commonly used to produce pellets, tablets or beads for drug delivery (Bartkowiak and Hunkeler, 2001; Piyakulawat et al., 2007; Thommes and Kleinebudde, 2008).

Hyaluronic acid (HA) is a major constituent of the cartilage extracellular matrix. Its biological properties and rapid degradation makes $\mathrm{HA}$ an ideal candidate for the development of new biomaterials (Liao et al., 2005; Kim et al., 2007). Fibrinogen/fibrin was selected because of its adhesive properties. Moreover, various studies have reported the use of fibrin gel as an injectable scaffold for generating new cartilage matrix (Eyrich et al., 2007a, 2007b).

We performed preliminary studies with gels composed of only HA and fibrin. When the articular chondrocytes or other types of cells were encapsulated in this gel, within a few days we observed a drastic shrinkage of the scaffold. Indeed, these unpublished observations are in agreement with recently published data (Eyrich et al., 2007a). Therefore, to improve the quality of the hydrogel, we introduced carrageenan into the HA-fibrin gel. With this new gel composition, we observed no shrinking effects; on the contrary, the gel presented a good elasticity as well as a better stiffness. 

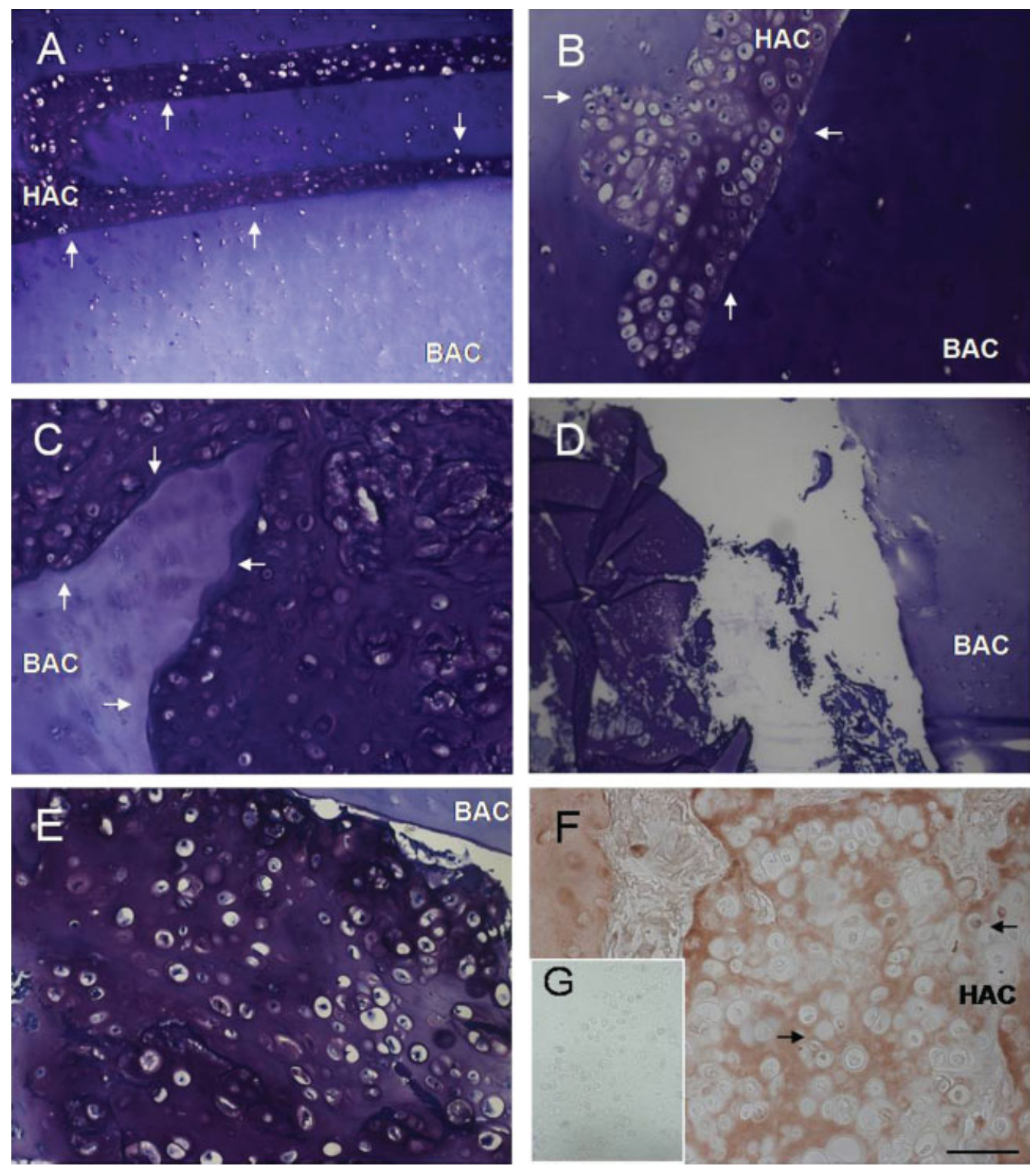

Figure 8. Histological cross-sections of the hydrogel within the bovine cartilage gap after 6 weeks from the implantation in immunodeficient mouse. Toluidine blue staining (A-E); immunohistochemical staining showing localization of type II collagen (F); negative control without primary antibody (G); control sample not seeded with cells (D). BAC, bovine articular cartilage; HAC, human articular cartilage. Bar $=50 \mu \mathrm{m}$. Arrows indicate the new human cartilage deposition and a good integration of the tissue

Chondrocytes suspended in a 3D matrix, similar to their natural environment, retain their native phenotype and organize a cartilage extracellular matrix. Hydrogel scaffolds appear to satisfy this requirement. We encapsulated human articular chondrocytes into this novel polysaccharide/fibrin hydrogel and cultured them in vitro for 3 weeks. Our data indicate that this injectable scaffold sustains an initial cell growth without compromising the cell chondrogenic potential.

The growth and the distribution of the cells within the hydrogel were qualitatively confirmed via images obtained by histology using brightfield and epifluorescent light microscopy. In particular, structured epifluorescent illumination and optical sectioning allowed the acquisition of images through most of the hydrogel thickness without the need of any manipulation, such as the dehydration required for histology that may introduce artifacts. This analysis revealed a uniform distribution of viable human articular chondrocytes throughout the hydrogel up to 3 weeks of culture.

After an encapsulation period of 3 weeks into the tissue-engineered construct, cells not only maintained the expression of proteins characteristic of the chondrogenic lineage but also downregulated the expression of type I collagen characteristically expressed by dedifferentiated articular chondrocytes and by articular chondrocytes expanded in monolayer culture in serum-free medium.

We are aware that chondrocytes harvested from osteoarthritic patients are not the best source of cells for cartilage regeneration. Chondrocytes harvested from healthy tissue or other cell populations, such as mesenchymal stem cells, may have led to even better results. 

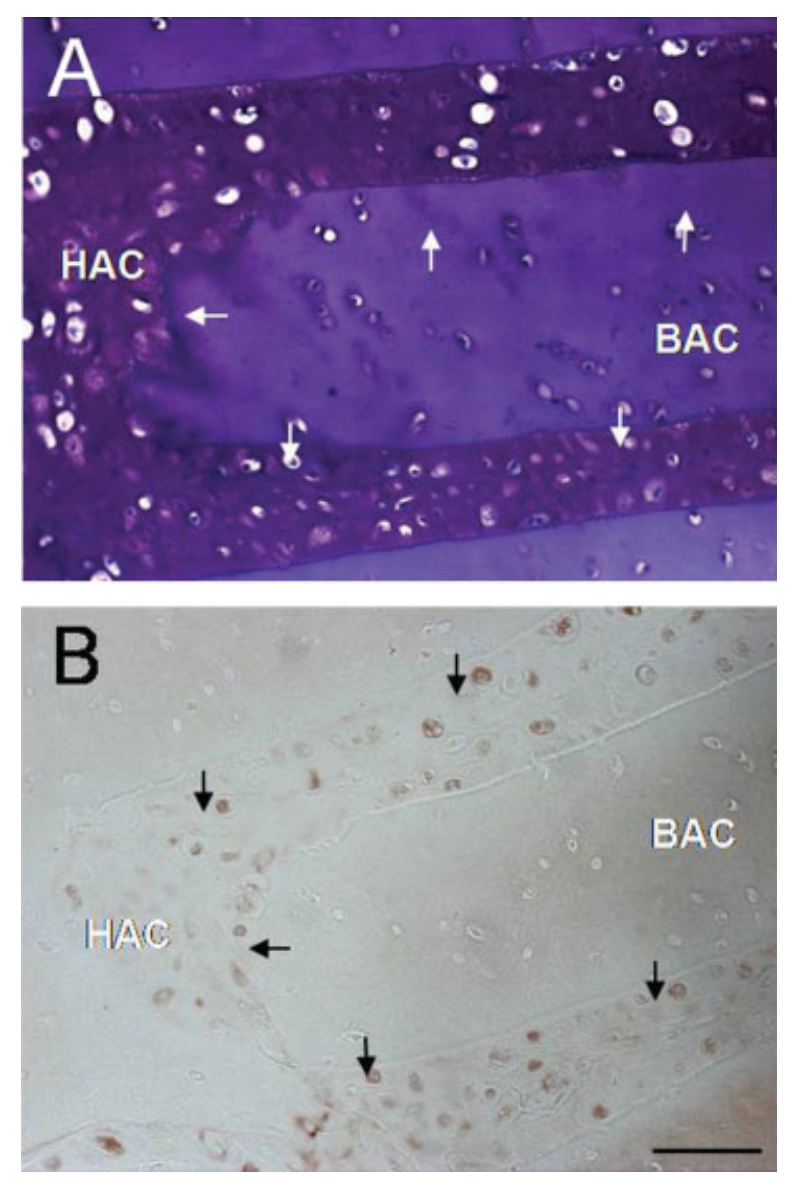

Figure 9. Histological cross-sections of the hydrogel within the bovine cartilage gap after 6 weeks from implantation in immunodeficient mouse. The neocartilage formed shows strong staining with toluidine blue (A). The specific anti-human tenascin antibody recognizes only chondrocytes in the newly formed cartilage in the gap defect (B). BAC, bovine articular cartilage; HAC, human articular cartilage. $B a r=50 \mu \mathrm{m}$. White arrows show the area of integration of human articular cartilage in the bovine tissue. Black arrows show the human chondrocyte cells

To provide a definitive assessment concerning the suitability of a new biomaterial for cartilage tissue engineering, in vivo models are required. The 'conventional' immunodeficient mouse model can support cartilage formation but fails in investigating integration of the newly formed cartilage within a pre-existing cartilage. Therefore, in our study we used a contained-defect organ culture model, recently described by Mueller-Rath et al. (2007) and Sculler et al. (2008). This experimental model mimics a real clinical intra-articular situation where the repair tissue is having direct contact with the surrounding cartilage. In view of the difficulties in harvesting human articular cartilage, we have been forced to used two different species to test the capability of human articular chondrocytes expanded in vitro to regenerate tissue in a bovine cartilage lesion.

Using this approach, we observed a good integration between the human cartilaginous tissue formed in the articular cartilage defect and the surrounding bovine cartilage after 6 weeks from the implantation.

\section{Conclusions}

In this study, we demonstrated the suitability for cartilage engineering of a newly developed injectable gel. Within this novel hydrogel, encapsulated human articular chondrocytes remained viable, proliferated, maintained the expression of the typical chondrogenic marker genes and deposited a cartilage ECM. Taking advantage of a new in vivo model, we showed the integration of the newly formed cartilage with the surrounding preexisting cartilage. We propose that the novel hydrogel we developed may be used with success to deliver ex vivo expanded articular chondrocytes by arthroscopy injection to the site of the articular cartilage lesion, and therefore to repair the articular cartilage damage using a minimally invasive technique.

\section{Acknowledgements}

The authors are grateful to Recco orthopaedic staff members for the collaboration and patients for bioptic material donation as well as to Mrs Daniela Marubbi for histological assistance. This work was supported by funds from the Italian MUR (FIRBTissuenet project), the European Union-funded STREP project, HIPPOCRATES (Grant No. NMP3-CT-2003-505758) and the European NoE EXPERTISSUES project (Grant No. NMP3-CT2004-500283).

\section{References}

Ando W, Tateishi K, Hart DA, et al. 2007; Cartilage repair using an in vitro generated scaffold-free tissue-engineered construct derived from porcine synovial mesenchymal stem cells. Biomaterials 28(36): 5462-5470.

Banfi A, Muraglia A, Dozin B, et al. 2000; Proliferation kinetics and differentiation potential of ex vivo expanded human bone marrow stromal cells: implications for their use in cell therapy. Exp Hematol 28(6): 707-715.

Bartkowiak A, Hunkeler D. 2001; Carrageenan-oligochitosan microcapsules: optimization of the formation process (1). Colloids Surf B Biointerfaces 21(4): 285-298.

Brittberg M, Lindahl A, Nilsson A, et al. 1994; Treatment of deep cartilage defects in the knee with autologous chondrocyte transplantation. N Engl J Med 331(14): 889-895.

Cancedda R, Dozin B, Giannoni P, et al. 2003; Tissue engineering and cell therapy of cartilage and bone. Matrix Biol 22(1): 81-91.

Chung C, Burdick JA. 2008; Engineering cartilage tissue. Adv Drug Deliv Rev 60(2): 243-262.

DeFail AJ, Chu CR, Izzo N, et al. 2006; Controlled release of bioactive $\mathrm{TGF} \beta 1$ from microspheres embedded within biodegradable hydrogels. Biomaterials 27(8): 1579-1585.

Denizot F, Lang R. 1986; Rapid colorimetric assay for cell growth and survival. Modifications to the tetrazolium dye procedure giving improved sensitivity and reliability. J Immunol Methods 89(2): 271-277.

Dozin B, Malpeli M, Camardella L, et al. 2002; Response of young, aged and osteoarthritic human articular chondrocytes to inflammatory cytokines: molecular and cellular aspects. Matrix Biol 21(5): 449-459.

Endres M, Neumann K, Schroder SE, et al. 2007; Human polymerbased cartilage grafts for the regeneration of articular cartilage defects. Tissue Cell 39(5): 293-301.

Eyrich D, Brandl F, Appel B, et al. 2007a; Long-term stable fibrin gels for cartilage engineering. Biomaterials 28(1): 55-65.

Eyrich D, Wiese H, Maier G, et al. 2007b; In vitro and in vivo cartilage engineering using a combination of chondrocyte-seeded longterm stable fibrin gels and polycaprolactone-based polyurethane scaffolds. Tissue Eng 13(9): 2207-2218. 
Frey MR, , Dise RS, Edelblum KL, et al. 2006; p38 kinase regulates epidermal growth factor receptor downregulation and cellular migration. EMBO J 25(24): 5683-5692.

Gelse K, Schneider H. 2006; Ex vivo gene therapy approaches to cartilage repair. Adv Drug Deliv Rev 58(2): 259-284.

Giannoni P, Cancedda R. 2006; Articular chondrocyte culturing for cell-based cartilage repair: needs and perspectives. Cells Tissues Organs 184(1): 1-15.

Gutowska A, Jeong B, Jasionowski M, et al. 2001; Injectable gels for tissue engineering. Anat Rec 263(4): 342-349.

Hunziker EB. 1999; Biologic repair of articular cartilage. Defect models in experimental animals and matrix requirements. Clin Orthop Relat Res 367: (suppl): S135-146.

Hunziker EB. 2000; Articular cartilage repair: problems and perspectives. Biorheology 37(1-2): 163-164.

Johnstone B, Hering TM, Caplan AI, et al. 1998; In vitro chondrogenesis of bone marrow-derived mesenchymal progenitor cells. Exp Cell Res 238(1): 265-272.

Kim J, Kim IS, Cho TH, et al. 2007; Bone regeneration using hyaluronic acid-based hydrogel with bone morphogenic protein2 and human mesenchymal stem cells. Biomaterials 28(10): 1830-1837.

Lee KY, Mooney DJ. 2001; Hydrogels for tissue engineering. Chem Rev 101(7): 1869-1879.

Liao YH, Jones SA, Forbes B, et al. 2005; Hyaluronan: pharmaceutical characterization and drug delivery. Drug Deliv 12(6): 327-342.

Liu W, Cao Y. 2007; Application of scaffold materials in tissue reconstruction in immunocompetent mammals: our experience and future requirements. Biomaterials 28(34): 5078-5086.

Malpeli M, Randazzo N, et al. 2004; Serum-free growth medium sustains commitment of human articular chondrocyte through maintenance of Sox9 expression. Tissue Eng 10(1-2): 145-155.

McHale MK, Setton SA, Chilkoti A, et al. 2005; Synthesis and in vitro evaluation of enzymatically cross-linked elastin-like polypeptide gels for cartilaginous tissue repair. Tissue Eng 11(11-12): 1768-1779.

Mueller-Rath R, Gavenis K, Gravius S, et al. 2007; In vivo cultivation of human articular chondrocytes in a nude mouse-based contained defect organ culture model. Biomed Mater Eng 17(6): 357-366.
Neuss S, Apel C, Buttler P, et al. 2008; Assessment of stem cell/biomaterial combinations for stem cell-based tissue engineering. Biomaterials 29(3): 302-313.

Osakada F, Ikeda H, Mandai M, et al. 2008; Toward the generation of rod and cone photoreceptors from mouse, monkey and human embryonic stem cells. Nat Biotechnol 26(2): 215-224.

Park H, Temenoff JS, Holland TA, et al. 2005; Delivery of TGF- $\beta 1$ and chondrocytes via injectable, biodegradable hydrogels for cartilage tissue engineering applications. Biomaterials 26(34): 7095-7103.

Park H, Temenoff JS, Tabata Y, et al. 2007; Injectable biodegradable hydrogel composites for rabbit marrow mesenchymal stem cell and growth factor delivery for cartilage tissue engineering. Biomaterials 28(21): 3217-3227.

Peterson L, Minas T, Brittberg M, et al. 2000; Two- to 9-year outcome after autologous chondrocyte transplantation of the knee. Clin Orthop Relat Res 374: 212-234.

Piyakulawat P, Praphairaksit N, Chantarasiri N, et al. 2007; Preparation and evaluation of chitosan/carrageenan beads for controlled release of sodium diclofenac. AAPS PharmSciTech 8(4): E97.

Schuller GC, Tichy B, Majdisova Z, et al. 2008; An in vivo mouse model for human cartilage regeneration. $J$ Tissue Eng Regen Med 2(4): 202-209.

Temenoff JS, Mikos AG. 2000a; Injectable biodegradable materials for orthopedic tissue engineering. Biomaterials 21(23): 2405-2412.

Temenoff JS, Mikos AG. 2000b; Review: tissue engineering for regeneration of articular cartilage. Biomaterials 21(5): 431-440.

Thommes M, Kleinebudde P. 2008; The behavior of different carrageenans in pelletization by extrusion/spheronization. Pharm Dev Technol 13(1): 27-35.

Tuli R, Li W, Tuan RS, et al. 2003; Current state of cartilage tissue engineering. Arthritis Res Ther 5(5): 235-238.

Ulivi V, Tutolo G, Mallein-Gerin F, et al. 2006; A common pathway in differentiation and inflammation: p38 mediates expression of the acute phase SIP24 iron binding lipocalin in chondrocytes. $J$ Cell Physiol 206(3): 728-737. 